\title{
Over-expression of cyclin B1 in human endometrial cancer.
}

Shahan Mamoor, $\mathrm{MS}^{1}$

${ }^{1}$ shahanmamoor@gmail.com

4 East Islip, NY USA

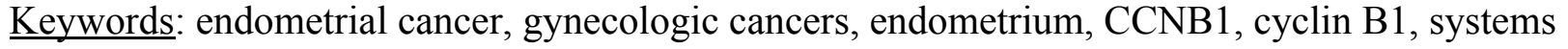
biology of endometrial cancer, targeted therapeutics in endometrial cancer.

Gynecologic cancers including cancers of the endometrium are a clinical problem ${ }^{1-4}$. We mined published microarray data ${ }^{5,6}$ to discover genes associated with endometrial cancers by comparing transcriptomes of the normal endometrium and endometrial tumors from humans. We identified the cell cycle component cyclin B1, encoded by CCNB1, as among the most differentially expressed genes, transcriptome-wide, in cancers of the endometrium. CCNB1 was expressed at significantly higher levels in endometrial tumor tissues as compared to the endometrium. Importantly, high primary tumor expression of CCNB1 was correlated with worse overall survival in patients with endometrial cancer. Together, the data reveal over-expression of CCNB1 in human endometrial cancer and describe specific activation of the cell cycle in cancers of the endometrium. 
Endometrial cancer is the most common gynecologic cancer in the developed world ${ }^{1}$. Over the last three decades, the incidence of endometrial cancer has increased $21 \%{ }^{4}$ and the death rate has increased $100 \%{ }^{3}$. We harnessed the power of independently published microarray datasets $^{5,6}$ to determine in an unbiased fashion and at the systems-level genes most differentially expressed in endometrial tumors. We report here the differential and increased expression of the Cyclin B1 (CCNB1) in human endometrial cancer.

\section{Methods}

We utilized datasets GSE63678 5 and GSE $17025^{6}$ for this global differential gene expression analysis of human endometrial cancer in conjunction with GEO2R. GSE63678 was generated using Affymetrix Human Genome U133A 2.0 Array technology with $n=5$ control endometrial tissues (including $n=4$ uterine myomas and $n=1$ benign cyst) and $n=7$ endometrial cancers (including $n=2$ endometrial adenocarcinomas, $n=3$ mixed endometrioid adenocarcinomas, and $n=2$ adenocarcinomas with squamous differentiation); analysis was performed using platform GPL571. GSE17025 was generated using Affymetrix Human Genome U133 Plus 2.0 Array technology with $n=5$ normal endometrial tissues (inactive endometrium) and $n=91$ endometrial cancers; analysis was performed using platform GPL570. The Benjamini and Hochberg method of $p$-value adjustment was used for ranking of differential expression but raw $p$-values were used to assess statistical significance of global differential expression.

Log-transformation of data was auto-detected, and the NCBI generated category of platform annotation was used. A statistical test was performed to evaluate whether CCNB1 gene expression was significantly different between primary tumors of the breast and brain metastases in humans with breast cancer using a two-tailed t-test. For Kaplan-Meier survival analysis, we used the Kaplan-Meier plotter tool ${ }^{7}$ for correlation of CCNB1 mRNA expression levels with overall survival in $n=543$ endometrial cancer patients.

\section{$\underline{\text { Results }}$}

We harnessed the power of blind comparative transcriptome analysis using published microarray data ${ }^{5,6}$ to discover in an unbiased fashion genes associated with endometrial cancer in humans.

\section{CCNB1 is differentially expressed in endometrial cancer.}

We identified cyclin B1, encoded by CCNB1, as among the genes most differentially expressed in cancers of the endometrium when compared to benign endometrial tissues (Chart 1). When sorting each of the genes expressed in endometrial tumor tissue based on significance of change in expression as compared to benign endometrial tissue, CCNB1 ranked 8 out of 22273 transcripts, equating to $99.96 \%$ differential expression (Chart 1). Differential expression of CCNB1 in human endometrial cancers was statistically significant (Chart $1 ; p=6.48 \mathrm{e}-06$ ). 
We queried a second microarray data to validate differential expression of CCNB1 in

Thus, by mining published microarray data ${ }^{5,6}$ in an unbiased and systematic fashion, we identified Cyclin B1, encoded by CCNB1, as among the genes whose expression was most different, transcriptome-wide, in the endometrial tumor tissue of patients with endometrial cancer when compared to benign endometrial tissue; we observed significantly increased expression of CCNB1 in endometrial tumor tissue as compared to benign endometrial tissue. Further, we found a correlation between CCNB1 expression and patient survival outcomes in human endometrial cancer, as overall survival was greater in patients whose tumors expressed higher levels of CCNB1 as compared to patients whose tumors expressed lower levels of CCNB1. 


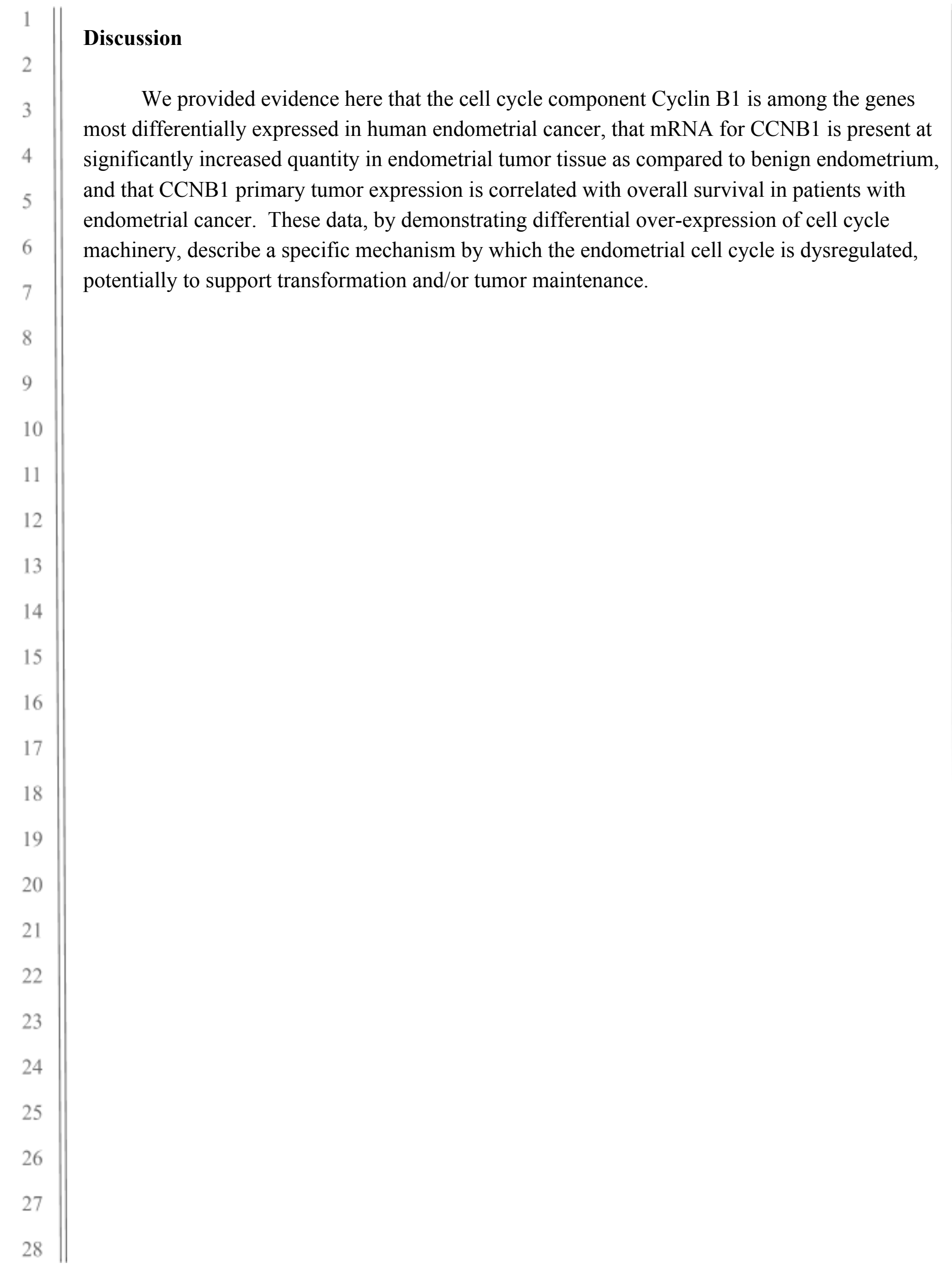




\section{References}

1. Amant, F., Moerman, P., Neven, P., Timmerman, D., Van Limbergen, E. and Vergote, I., 2005. Endometrial cancer. The Lancet, 366(9484), pp.491-505.

2. Sorosky, J.I., 2008. Endometrial cancer. Obstetrics \& Gynecology, 111(2), pp.436-447.

3. Morice, P., Leary, A., Creutzberg, C., Abu-Rustum, N. and Darai, E., 2016. Endometrial cancer. The Lancet, 387(10023), pp.1094-1108.

4. Sorosky, J.I., 2012. Endometrial cancer. Obstetrics \& Gynecology, 120 (2 Part 1), pp.383-397.

5. Pappa, K.I., Polyzos, A., Jacob-Hirsch, J., Amariglio, N., Vlachos, G.D., Loutradis, D. and Anagnou, N.P., 2015. Profiling of discrete gynecological cancers reveals novel transcriptional modules and common features shared by other cancer types and embryonic stem cells. PLoS One, 10(11), p.e0142229.

6. Day, R.S., McDade, K.K., Chandran, U.R., Lisovich, A., Conrads, T.P., Hood, B.L., Kolli, V.K., Kirchner, D., Litzi, T. and Maxwell, G.L., 2011. Identifier mapping performance for integrating transcriptomics and proteomics experimental results. BMC bioinformatics, 12(1), p.213.

7. Nagy, A., Munkacsy, G. and Gyorffy, B., 2020. Pancancer survival analysis of cancer hallmark genes. bioRxiv. 


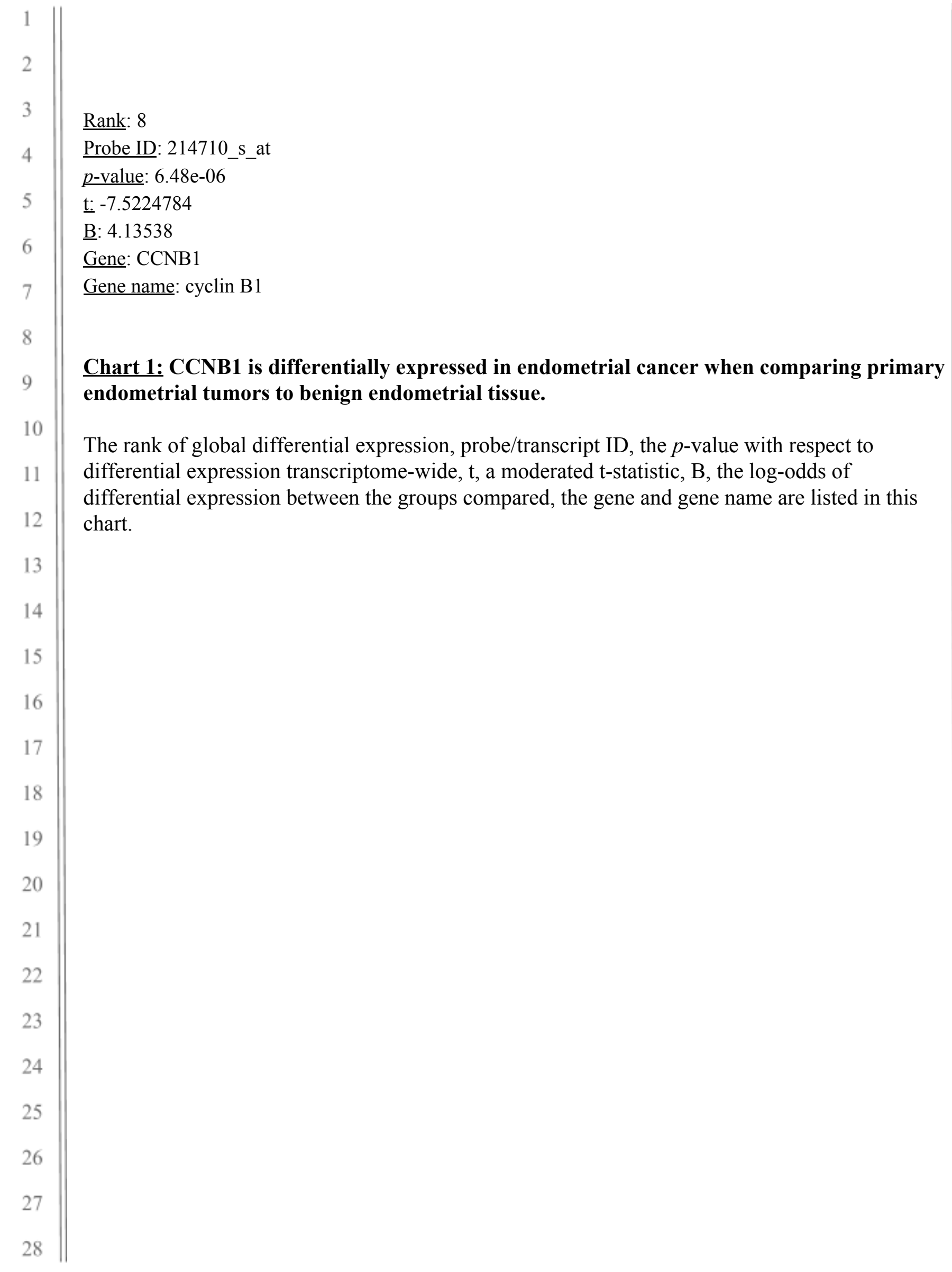




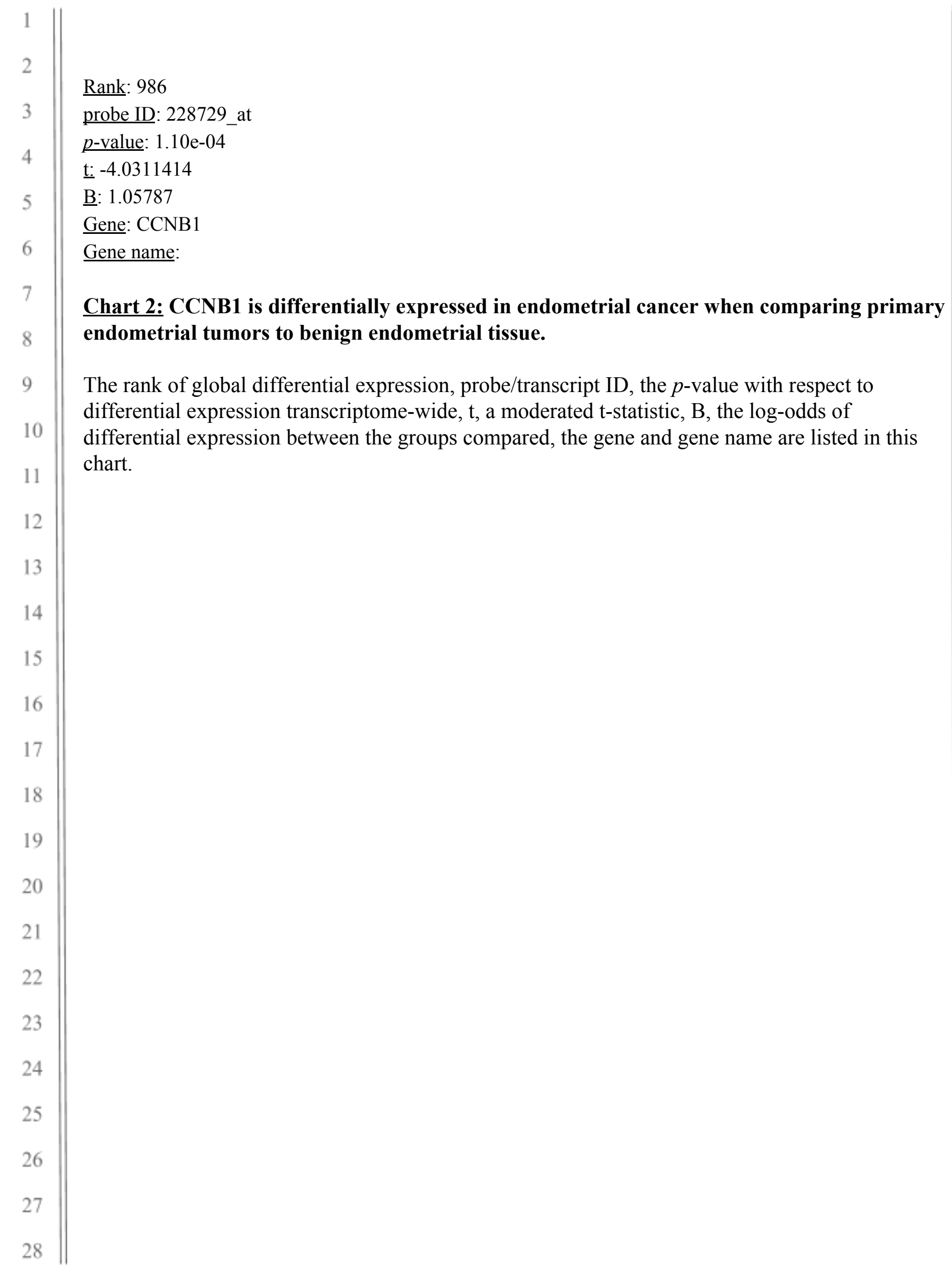




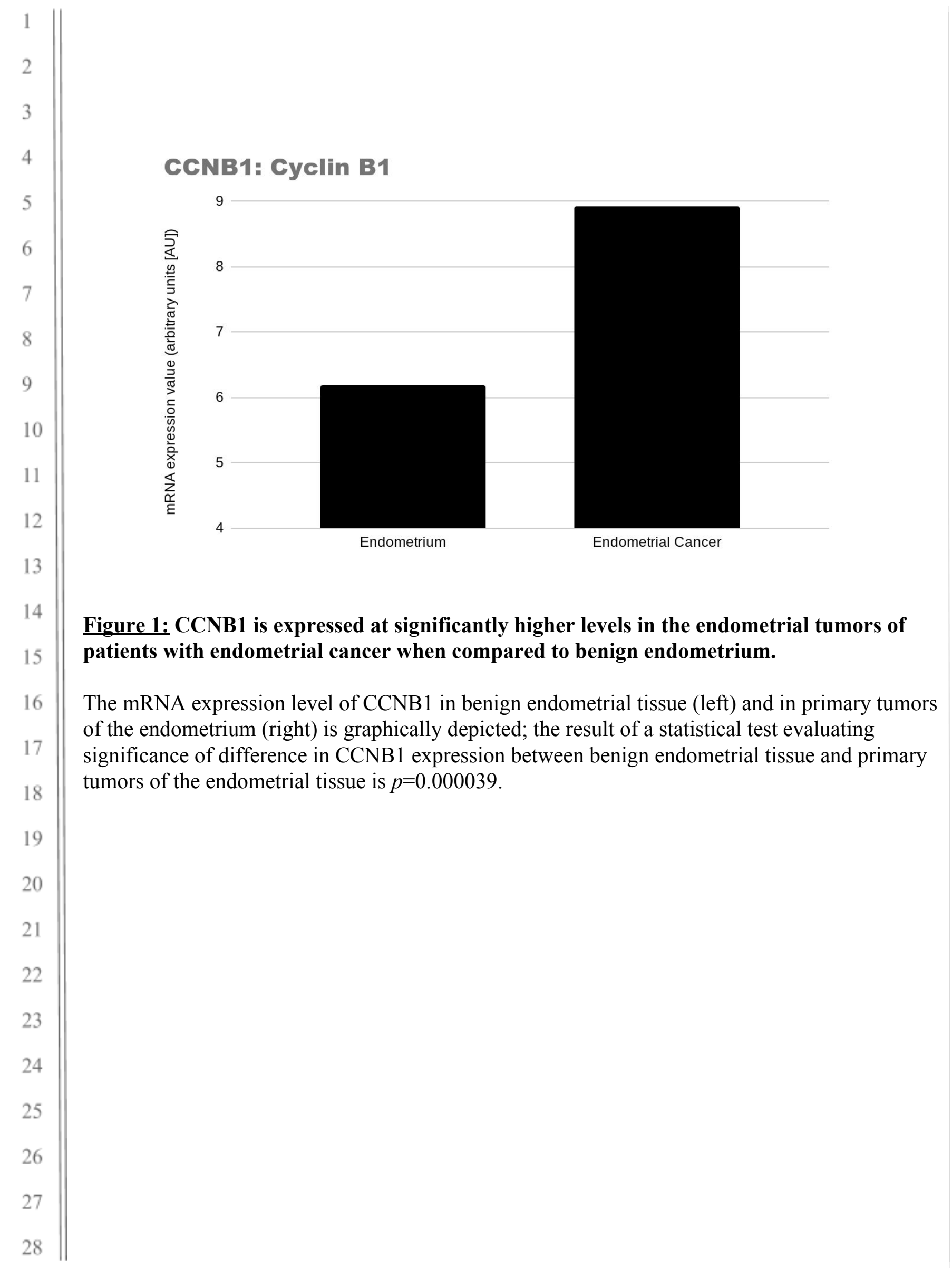




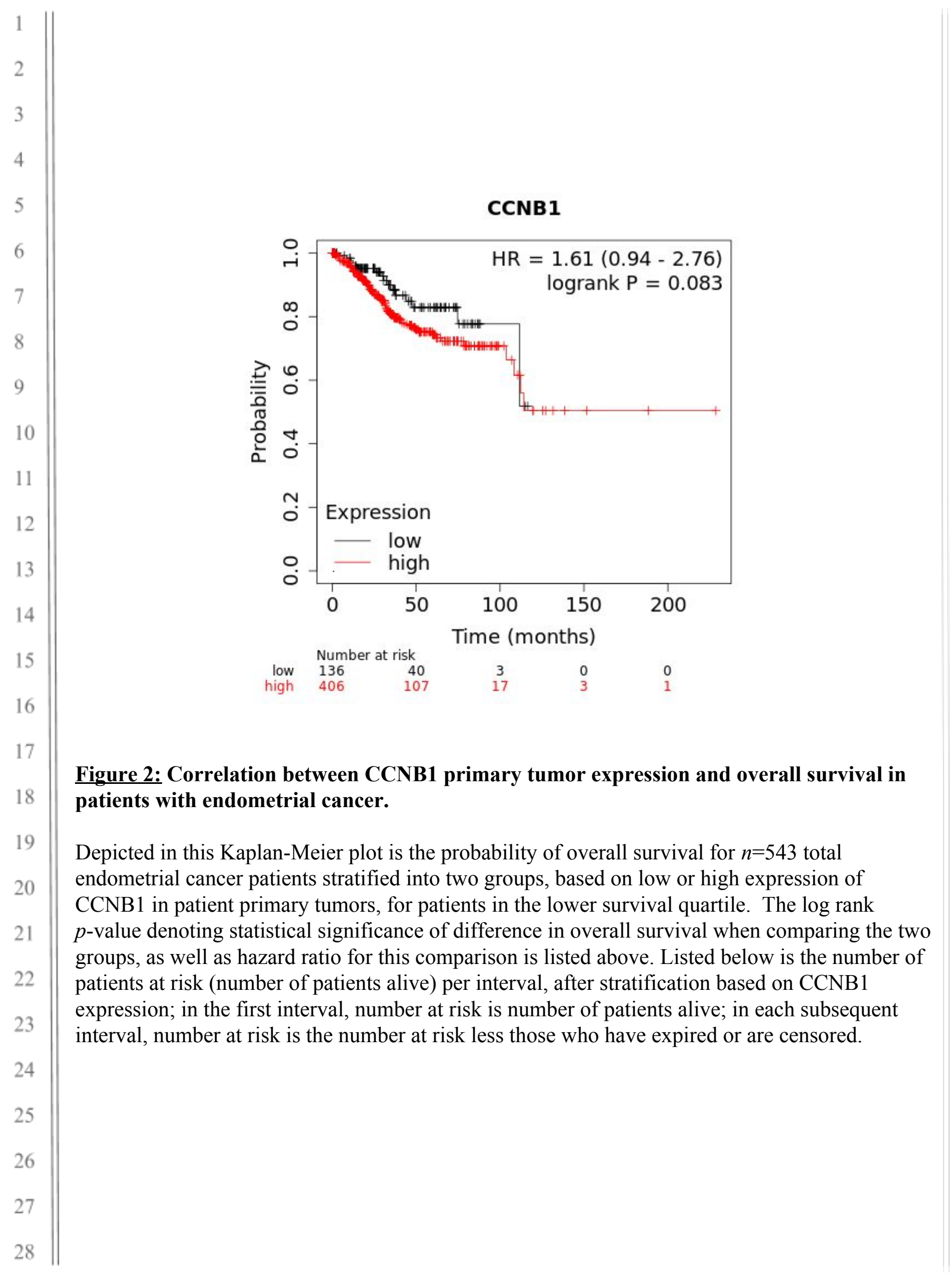


Low CCNB1 expression: 111.63 months

High CCNB1 expression: 59.77 months

Chart 3: Median overall survival is inferior in endometrial cancer patients with high primary tumor expression of CCNB1.

The median OS (overall survival) of patients with low primary tumor expression of CCNB1 and high primary tumor expression of CCNB1 is listed in this chart, for $n=543$ endometrial cancer patients, in the lower survival quartile. 